\title{
Tunnelling in Organic Superconductors
}

\author{
C. J. Bolech ${ }^{1,2}$ and T. Giamarchi ${ }^{1}$ \\ ${ }^{1}$ University of Geneva, 24 Quai Ernest Ansermet, 1211 Geneva, Switzerland \\ E-mail: thierry.giamarchi@physics.unige.ch \\ ${ }^{2}$ Rice University, 6100 Main Street, Houston, TX 77251-1892, USA
}

We present a simple scheme for computing the full current-voltage characteristics for tunnelling experiments within the framework of the non-equilibrium Keldysh Green function formalism. This formalism is flexible enough to address different pairing symmetries combined with magnetic fields at arbitrary bias voltages. We show how to apply these results to probe for the symmetry of the superconducting order parameter in the Bechgaard salts using tunnelling experiments.

KEY WORDS: organic superconductors; tunnel effect; triplet super conductivity.

\section{INTRODUCTION}

Organic materials have provided physicists with an extraordinary laboratory to study the effects of interactions in solids. ${ }^{1}$ Indeed such compounds made of coupled chains allow to realize Luttinger liquids, ${ }^{2}$ one of the very few controlled examples of non-Fermi liquids due to interactions. In addition, contrarily to other realizations of one dimensional systems, the organics offer unique challenges: because of their very three dimensional nature, they provide not a single one dimensional electron gas, but a very large number of such one dimensional systems coupled together. This allows thus for a unique new physics to emerge where the system is able to crossover from a one dimensional behavior to a more conventional three dimensional one.

As a consequence of this dimensional crossover, at low temperature these materials undergo instabilities towards three-dimensionally ordered states, such as spin-Peierls, antiferromagnetic and even superconducting states. Needless to say, the presence of superconductivity in these compounds is a tantalizing and challenging question. Despite the period of 
a quarter of a century since the discovery of superconductivity in these materials, ${ }^{3}$ the mechanism and even the symmetry of this superconducting phase have remained elusive, and many efforts have been devoted to this subject. Recently, the case for triplet nature of this superconducting phase has been made, ${ }^{4}$ mostly by measurements of the upper critical field and by NMR measurements, but the subject is far from being settled.

We explain here how point contact tunnelling experiments can be used to probe for the symmetry of a superconducting state. In the recent years, the possibilities to perform point contact tunnelling have been drastically enhanced with the development of scanning tunnelling microscopy (STM). ${ }^{5}$ Correspondingly, theories to interpret tunnelling experiments in superconductors have evolved from simple semiconducting band models, ${ }^{6,7}$ to more systematic approaches based on the tunnelling Hamiltonian. ${ }^{8-10}$ We show here how one can extend and simplify the formalism to make it more versatile and easy to implement. ${ }^{11,12}$ This allows to study the case of superconducting (singlet or triplet) leads, as well as the effects of magnetic fields on the junction. The resulting theory can be thus directly used as a probe of the symmetry of the leads.

\section{TUNNELLING}

We model the system with a (one-dimensional) Hamiltonian that includes the two leads and a tunnelling term: $H=H_{1}+H_{2}+H_{\text {tun }}$. Each lead is described by

$$
K=\xi_{c k \sigma} \psi_{c k \sigma}^{\dagger} \psi_{c k \sigma}-\left\{\Delta_{a}\left[\psi_{R k \beta}^{\dagger} \sigma_{\beta \alpha}^{a} \alpha \psi_{L \bar{k} \bar{\alpha}}^{\dagger}\right]+\text { h.c. }\right\},
$$

where $K=H-\mu N$ and $\mu$ is the corresponding electrochemical potential. All the indices are summed over, in particular $k$ is the lattice momentum, Greek indices correspond to the spin, and $c \in(L, R) \equiv(-1,+1)$ sums over the two possible chiralities. $\xi_{c k \sigma}=c v_{\mathrm{F}} k-\mu-\sigma h$ are the corresponding linear dispersions, shifted by the inclusion of chemical potential and magnetic field along the $\hat{z}$-axis (for convenience we will take $v_{\mathrm{F}}=1$ ). This is the extension of the pairing-approximation Hamiltonian found in BCS theory to the triplet case. The third term in the Hamiltonian describes the tunnelling: $H_{\text {tun }}=t\left(\psi_{1 \sigma}^{\dagger}(0) \psi_{2 \sigma}(0)+\right.$ h.c. $)$. We define the current as given by the rate of change in the relative particle number caused by tunnelling: $I=\frac{e}{2 i}\left\langle\left[H_{\text {tun }}, N_{1}-N_{2}\right]\right\rangle$. It is justified, for simple superconductors, to use one-dimensional leads to carry out all the standard calculations. For unconventional superconductors the situation is more complex, because the anisotropic nature of the pair wave-function has to be taken into 
account when modelling the leads. But the organic superconductors are supposed to have $p$-wave symmetry and be highly anisotropic. We can therefore, as a first approximation, conveniently restrict ourselves to a onedimensional model and adopt a formalism that encompasses both $s$ - and $p$-wave symmetries, as well as the normal state. In order to deal with an out of equilibrium situation, we use the so-called Keldysh formalism. ${ }^{13}$ We treat the tunnelling term to all orders to calculate the full $\mathrm{I}-\mathrm{V}$ line and give a quantitative account of the subgap structure. We skip here all technical details and refer the reader to Ref. 11,12 for those.

\section{TEST FOR TRIPLET}

We choose here for illustration some convenient set of parameters. The tunnelling overlap integral takes the values $t=0.2$ and $t=0.5$, and, when present, a magnetic field of $h=0.2$ in units of $\Delta(\Delta$ is the magnitude of the singlet gap, $\Delta_{0}$, or of the triplet vector order parameter depending on the case). We show curves for the dc response in the limit of vanishing temperatures. The geometry of the junction corresponds to tunnelling perpendicular to the chains of the quasi-one-dimensional compound, with an orbital order parameter aligned along them. In this situation no mid-gap states $^{14}$ are expected and, therefore, no midgap features either. We concentrate on what happens at the conduction edge, in particular the effects of applied fields. We refer the reader to the literature for some recent studies that look at the effect of fields on zero bias anomalies. ${ }^{15}$

The case of normal-metal-superconductor junctions corresponds to standard STM experiments. Figure 1(a) shows typical curves for a pointcontact junction between a normal metal and a conventional singlet-pairing superconductor. The solid lines correspond to the $\mathrm{N}-\mathrm{S}$ junction in zero field and the dashed line is for one of the junctions in the presence of a magnetic field that produces what would be seen as a Zeeman splitting of the differential conductance peak. The second part of the figure corresponds to a junction between a normal metal and an unconventional triplet-pairing superconductor. The solid lines correspond to the $\mathrm{N}-\mathrm{T}$ junction in zero field and the dashed line is for the $t=0.2$ junction when in the presence of a magnetic field aligned with the vector order parameter $\vec{\Delta}$. If one considers a magnetic field perpendicular to the order parameter $(\vec{h} \perp$ $\vec{\Delta}$ ), it has no effect on the I-V characteristic.

Figure 2 corresponds to junctions in which both sides are conventional spin-singlet superconductors and junctions connecting a spin-singlet and spin-triplet superconductor. This corresponds to a potential STM experiment with a superconducting tip made out of a conventional superconductor used to probe a superconducting phase of unknown symmetry. 


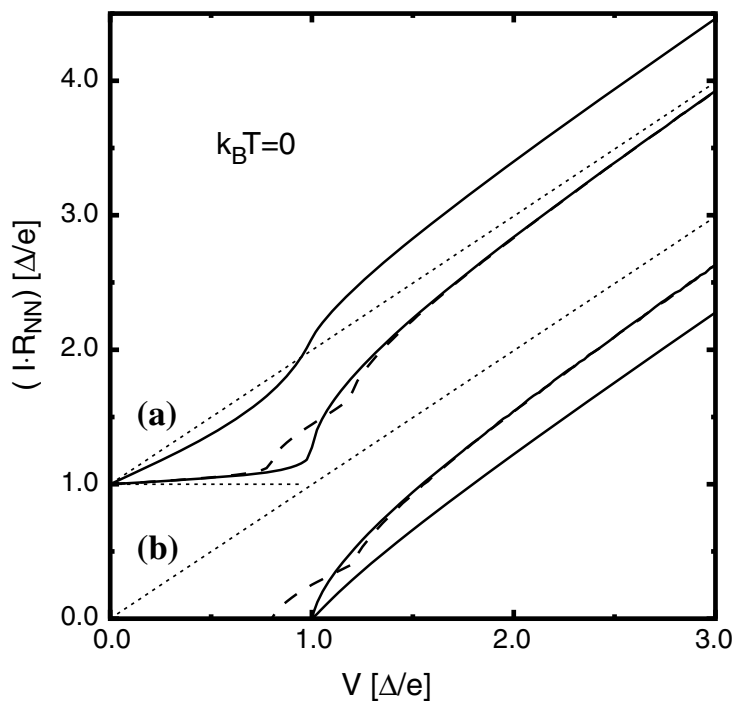

Fig. 1. I-V characteristics of normal-superconductor junctions. (a) $\mathrm{N}-\mathrm{S}$ junctions for $t=0.2$ (lower curves: with and without applied magnetic field, dashed and solid line respectively, $h=$ 0.2 ) and $t=0.5$ (upper curve, solid line only); the curves are vertically displaced for clarity. (b) $\mathrm{N}-\mathrm{T}$ junctions for $t=0.2$ (upper curves) and $t=0.5$ (lower curve).

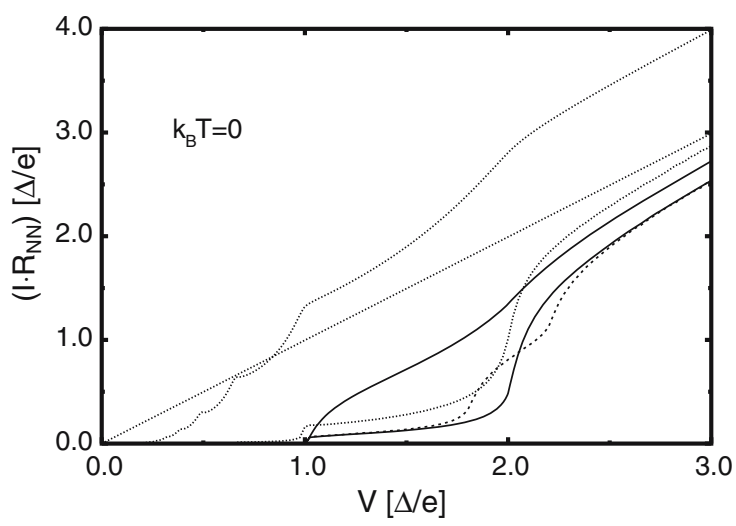

Fig. 2. I-V characteristics of $\mathrm{S}-\mathrm{T}$ junctions with and without magnetic field (dashed and solid lines respectively, $h=0.2$ ). The lower solid and dashed curves are for $t=0.2$ (without and with magnetic field) and the upper solid curve is for $t=0.5$. The dotted lines are (i) the straight unitary slope line given for reference and (ii) the S-S characteristics for similarparameters junctions (upper $t=0.5$ and lower $t=0.2$ ) given for comparison purposes.

In the case of conventional superconductors (dotted lines) and when orbital effects can be ignored, the $\mathrm{I}-\mathrm{V}$ is not sensitive to applied magnetic fields. On the other hand, the solid lines correspond to singlet-triplet 
junctions, that are insensitive to the orientation of the vector order parameter on the triple-pairing side of the junctions, and their current amplitude is found to be systematically smaller than in the case of the respective singlet-singlet junctions. The 'sub-gap' structure shows only two steps and the current is zero when $e V<\Delta_{\text {Triplet }}$ (the vector order parameter on the spin-triplet side of the junction). Concerning the effects of an applied magnetic field, the curves remain unchanged if the field is applied parallel to the direction of the vector-order-parameter, but show instead a Zeeman effect if the field is perpendicular to it (dashed line).

In the organics ${ }^{1}$ the experiments show that for magnetic fields along the direction of the conducting chains (a) the upper critical field is possibly paramagnetically limited for small fields (before crossing the upper critical field along $\mathbf{b}^{\prime}$ that is never paramagnetically limited). In that range we could assume that the direction of the order parameter is fixed respect to the lattice and does not follow the applied field. ${ }^{16}$ With this geometry, a Zeeman splitting of the differential conductance peak should be observed in a normal-tip STM experiment. As the field is rotated the splitting would be suppressed and for a magnetic field oriented parallel to the $\mathbf{b}^{\prime}$ crystalline-axis there should be no Zeeman effect. The disappearance of splitting, even as the field is possibly being increased, would constitute a clear signature of spin-triplet superconductivity. Similarly, an $s$-wave-tip STM would also be a direct probe for spin-triplet order. When a field is applied along the $\mathbf{b}^{\prime}$ crystalline-axis, a Zeeman splitting would occur. This would constitute a clear sign of unconventional superconductivity since such an effect does not take place for standard BCS superconductors. The $\mathbf{b}^{\prime}$ direction is the one on which the upper critical field is not paramagnetically limited, so relatively large fields could be applied in order to obtain a clear signal, and as the field alignment changes the splitting should disappear. No successful attempts of this kind of experiments were as yet made in the case of the quasi-one-dimensional organic salts, but efforts in this direction are on their way. Recently, preliminary experiments involving junctions between two Bechgaard salts were performed, and they showed a number of puzzling features including a zero-bias conductance peak and zero excess current. ${ }^{17}$ We certainly hope that further tunnelling experiments along the lines described on these notes can be performed.

\section{ACKNOWLEDGMENTS}

We would like to thank Ø. Fischer, M. Eskildsen, M. Kugler, and G. Levy for discussions about tunneling and STM. We also thank Y. Maeno and M. Sigrist for discussions about tunneling into triplet superconductors and ruthenates in particular. Part of this work has been 
supported by the Swiss National Science Fundation under MaNEP and Division II.

\section{REFERENCES}

1. 2004, for recent reviews on organics, see the volume 104 of Chemical Reviews.

2. T. Giamarchi, Quantum Physics in One Dimension (Oxford University Press, Oxford, 2004).

3. D. Jérome, A. Mazaud, M. Ribault, and K. Bechgaard, J. Phys. Lett. 41, L (1980).

4. T. Ishiguro, in High Magnetic Fields: Applications in Condensed Matter Physics and Spectroscopy, Vol. 595 of Lecture Notes in Physics, edited by C. Berthier, L. P. Lévy, and G. Martinez (Springer-Verlag, Heidelberg, 2002), pp. 301-313.

5. G. Binnig and H. Rohrer, Rev. Mod. Phys. 71, S324 (1999).

6. G. E. Blonder, M. Tinkham, and T. M. Klapwijk, Phys. Rev. B 25, 4515 (1982).

7. M. Octavio, M. Tinkham, G. E. Blonder, and T. M. Klapwijk, Phys. Rev. B 27, 6739 (1983).

8. M. H. Cohen, L. M. Falicov, and J. C. Phillips, Phys. Rev. Lett. 8, 316 (1962).

9. J. W. Wilkins, in Tunneling Phenomena in Solids, Multiparticle Tunneling, 1st ed. (Plenum Press, New York, 1969), chapter 24, p. 333.

10. J. C. Cuevas, A. Martín-Rodero, and A. L. Yeyati, Phys. Rev. B 54, 7366 (1996).

11. C. J. Bolech and T. Giamarchi, Phys. Rev. Lett. 92, 127001 (2004).

12. C. J. Bolech and T. Giamarchi, Phys. Rev. B 71, 024517 (2005).

13. L. V. Keldysh, Sov. Phys. JETP 24, 1018 (1965).

14. K. Sengupta et al., Phys. Rev. B 63, 144531 (2001).

15. Y. Tanuma et al., Phys. Rev. B 66, 094507 (2002).

16. A. G. Lebed, K. Machida, and M. Ozaki, Phys. Rev. B 62, R795 (2000).

17. H. I. Ha, J. I. Oh, J. Moser, and M. J. Naughton, Synth. Met. 137, 1215 (2003). 